\title{
Functional diversification of yeast telomere associated protein, Rif1, in higher eukaryotes
}

\author{
Easwaran Sreesankar ${ }^{1}$, Ramamoorthy Senthilkumar ${ }^{2}$, Vellaichamy Bharathi ${ }^{2}$, Rakesh K Mishra ${ }^{2 *}$ and \\ Krishnaveni Mishra ${ }^{*}$
}

\begin{abstract}
Background: Telomeres are nucleoprotein complexes at the end of linear eukaryotic chromosomes which maintain the genome integrity by regulating telomere length, preventing recombination and end to end fusion events. Multiple proteins associate with telomeres and function in concert to carry out these functions. Rap1 interacting factor 1 (Rif1), was identified as a protein involved in telomere length regulation in yeast. Rif1 is conserved upto mammals but its function has diversified from telomere length regulation to maintenance of genome integrity.

Results: We have carried out detailed bioinformatic analyses and identified Rif1 homologues in 92 organisms from yeast to human. We identified Rif1 homologues in Drosophila melanogaster, even though fly telomeres are maintained by a telomerase independent pathway. Our analysis shows that Drosophila Rif1 (dRif1) sequence is phylogenetically closer to the one of vertebrates than yeast and has identified a few Rif1 specific motifs conserved through evolution. This includes a Rif1 family specific conserved region within the HEAT repeat domain and a motif involved in protein phosphatase1 docking. We show that dRif1 is nuclear localized with a prominent heterochromatin association and unlike human Rif1, it does not respond to DNA damage by localizing to damaged sites. To test the evolutionary conservation of dRif1 function, we expressed the dRif1 protein in yeast and HeLa cells. In yeast, dRif1 did not perturb yeast Rif1 (yRif1) functions; and in HeLa cells it did not colocalize with DNA damage foci.
\end{abstract}

Conclusions: Telomeres are maintained by retrotransposons in all Drosophila species and consequently, telomerase and many of the telomere associated protein homologues are absent, including Rap1, which is the binding partner of Rif1. We found that a homologue of yRif1 protein is present in fly and dRif1 has evolutionarily conserved motifs. Functional studies show that dRif1 responds differently to DNA damage, implying that dRif1 may have a different function and this may be conserved in other organisms as well.

\section{Background}

Telomeres are nucleoprotein structures found at the ends of linear chromosomes and are critical for genome stability. In most eukaryotes, telomeric DNA consists of multiple copies of simple sequences ranging from a few hundred to a few thousand base pairs. These sequences are usually $G$ rich at the 3' end and are extended by a specialized, selftemplated reverse transcriptase, the telomerase. Telomeres play two important roles: (1) they serve as substrates for telomerase and thus prevent the loss of sequences at the very end as would be expected for a linear sequence replicated by semi-conservative DNA replication. This process is also

\footnotetext{
*Correspondence: mishra@ccmb.res.in; kms|@uohyd.ernet.in

${ }^{2}$ Centre for Cellular and Molecular Biology, Council of Scientific and Industrial Research, Uppal Road, Hyderabad 500 007, India

Full list of author information is available at the end of the article
}

precisely controlled in such a manner that only a designated amount of repeats are added and no uncontrolled elongation takes place. (2) They protect the ends from being recognized as double-strand breaks and from being attacked by nucleases. All these functions are carried out by multiple proteins that associate with the telomeres (reviewed in [1-4]).

Rif1 (Rap1 interacting factor) was identified in yeast Saccharomyces cerevisiae, as an interactor of the major telomere repeat sequence binding protein Rap1 [5]. Rif1 is a negative regulator of telomerase and together with another Rap1 interacting protein, Rif2, it controls the access of telomerase to telomere ends for replication and elongation of telomere sequences [6,7]. Accordingly, rif1 mutants have abnormally elongated telomeres. Furthermore, in the absence of telomerase, Rif1 inhibits the production of "Type

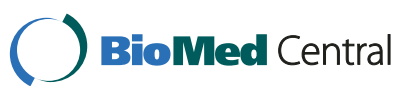


II" survivors, which use the Rad50 dependent recombination pathway to generate telomeres [8]. In yeast, Rif1 protein has been localized predominantly to telomeres where it also antagonizes the establishment of silent chromatin [911].

Given the key role of Rif1 in telomere biology, Rif1 homologues have been identified in other yeasts as well. In Schizosaccharomyces pombe, the Rif1 orthologue, is recruited to telomeres via another telomere sequence binding protein Taz1 and rif1 mutants have moderately elongated telomeres, suggesting that it is a negative regulator of telomere length [12]. However, as rif1 mutants in S. pombe show additive telomere length defects in rap1 mutants, it may work with Taz1 in a parallel pathway with Rap1 to control telomere length [13]. Furthermore, Rif1 has no effect on telomeric heterochromatin establishment in $S$. pombe. Recently, Rif1 orthologue from another budding yeast, Candida glabrata, has been studied. Although the effect on telomere length control by Rif1 was not reported, it was shown that in C. glabrata, Rif1 is essential for subtelomeric silencing [14]

Presence of Rif1 orthologues in vertebrates points to the key role of this protein in eukaryotes. Rif1 was first identified in mouse and was shown to be expressed at very high levels in totipotent and pluripotent cells, testes and was also associated with telomeres [15]. Subsequently, human Rif1 (hRif1) was identified and these studies suggested a divergence in the functions of Rif1 [16,17]. hRif1 associated with damaged DNA, including dysfunctional telomeres. Further studies established that hRif1 colocalized with several other DNA-damage response factors and depletion of hRif1 led to radiation sensitivity and defects in S-phase checkpoint. Additionally, through depletion studies in mouse cells, it has been demonstrated that mRif1 is essential and that it is involved in repair of stalled replication forks by homology directed repair [18]. hRif1 is upregulated in breast tumours and is proposed to be an anti-apoptotic factor required for DNA repair [19]. More recently, hRif1 was copurified with BLM helicase and was proposed to provide a DNA binding interface for recruiting factors involved in initiation of replication at stalled forks [20].

The studies from yeast to mammals show that Rif1 function has evolved from a protein that specifically participated in replication of the special DNA sequences present at the telomeres to a more general role in DNA damage response and reinitiation of replication at stalled replication forks. Drosophila, unlike mammals and yeasts, does not have simple sequence repeats at the telomeres. Instead they maintain their telomeres through the transposition of specialized non-LTR retroposons, namely, HeT-A, TART and TAHRE [21]. A putative Rif1 homologue in Drosophila has been reported based on sequence similarities to yeast Rif1 though its function has not been tested [12,16,20]. The presence of a Rif1 homologue in Drosophila suggests an early evolution of this telomeric protein to perform nontelomere related functions.

We performed a detailed bioinformatic analysis of Drosophila Rif1 (dRif1) to understand the evolutionary history of this protein. We found that Rif1 is conserved in all eukaryotes and dRif1 is closer to vertebrate Rif1 than yeast. A few conserved motifs were identified in the protein which will be helpful in elucidating the molecular basis of its function. We have followed the bioinformatic analyses with experimental test of conserved functions. We find that Drosophila and vertebrate Rif1 differ in their interaction with yeast telomeres and their response to DNA damage. Our data suggest that this protein has acquired additional domains in vertebrates and consequently additional roles.

\section{Results}

\section{Rif1 homologues are conserved across eukaryotes}

The Rif1 protein sequence of human and yeast were used for finding the homologues in NCBI protein sequence database. By this approach we found Rif1 homologues in 92 different organisms, including 54 fungal species, 18 insects and 16 vertebrate species (Additional file 1). In addition, we found the homologues in Hydra magnipapillata (Cnidarian), Trichoplax adhaerens (Placozoan) and Saccoglossus kowalevskii (Hemichordata). Phylogenetic tree constructed using the protein sequences of Rif1 shows an evolutionary pattern from lower to higher organisms (Figure 1A and Additional file 2) and indicates that the insect homologues are closer to human than fungal Rif1. We did not find clear homologues of Rif1 in plants, although a related protein in a lycophyte, Selaginella moellendorffii, was detected. While search with this lycophyte protein sequence in plants returned several uncharacterized proteins showing reasonable similarity (Additional file 3), these proteins lack the key conserved SILK/PP1 interaction domain (see below). We therefore deemed the plant homologues to be too diverged for further analysis.

\section{Conserved motifs in Rif1 homologues}

We found three motifs, namely, HEAT repeat, SILK motif and a domain present in the C-terminal end which was shown to have DNA binding property [20], that are conserved across the species from yeast to mammals in Rif1 (Figure 1B). In addition, previously predicted BLM helicase interaction domain is conserved only in the vertebrates [20]. HEAT repeat is a structural domain with poor sequence homology and is present in several proteins [22]. It spans 1000 amino acids in Rif1 homologues [20]. In our detailed analysis we found a highly conserved region of 101-149 amino acids present within the HEAT repeat that is Rif1 specific (Additional file 4). This domain is also present in the putative homologues identified in plants.

Our analysis identified another novel feature, SILK motif or Protein Phosphatase (PP1) interaction domain, in all 


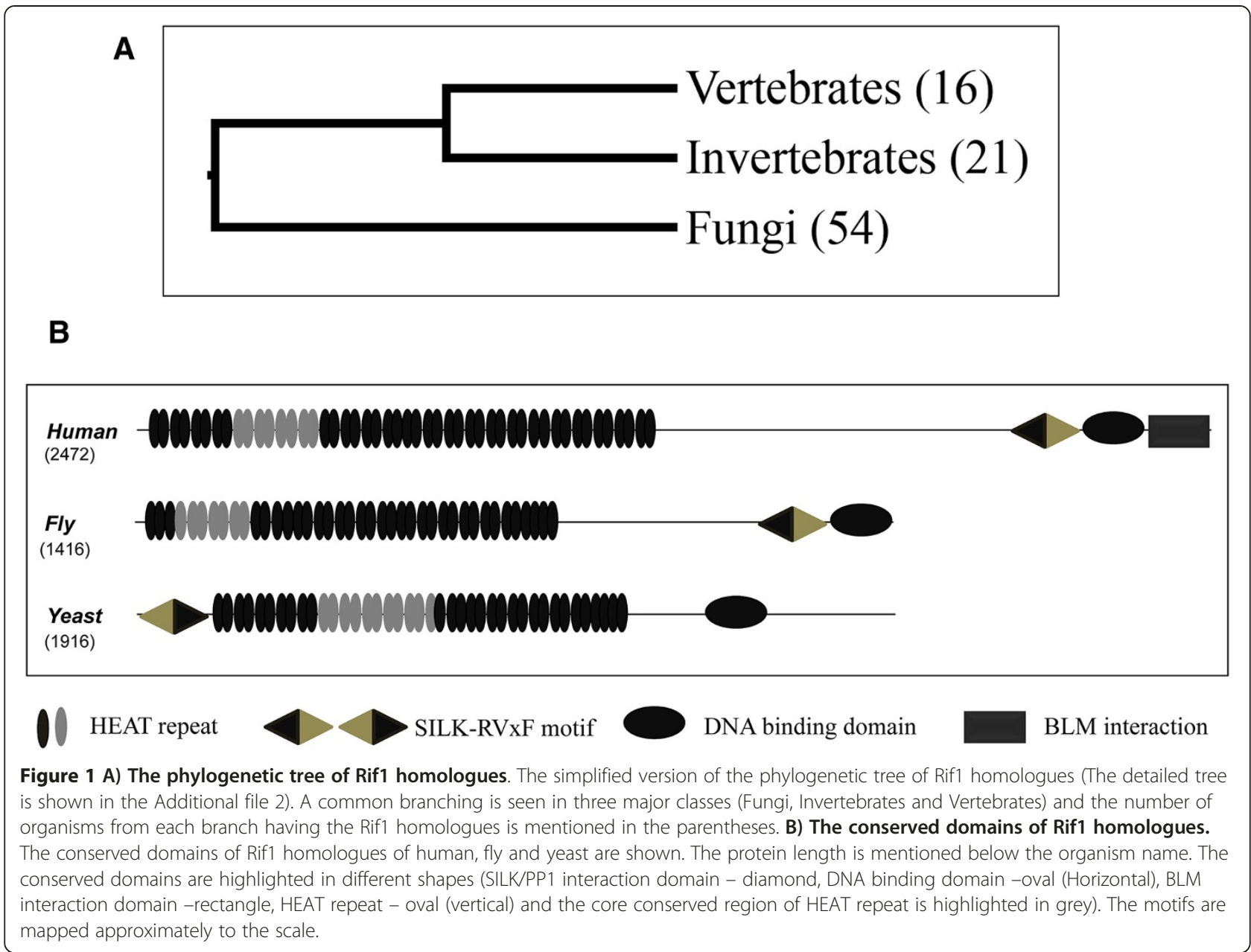

Rif1 homologues (Figure 1B, Additional files 5 and 6). The highly conserved residues $\mathrm{RVxF}$ were also detected along with the SILK motif, which is the docking motif essential for PP1 interaction [23,24]. Earlier studies have shown that the SILK motif is specifically associated with RVxF motif in certain class of PP1 interacting proteins $[23,25]$. In all Rif1 homologues, we found SILK and RVxF combination to be present with varying length of amino acid sequences between them. Recently, a large scale proteomics study revealed that the mammalian Rif1 interacts with PP1 by affinity chromatography [26], indicating that Rif1 is a target of PP1. Interestingly, the SILK-RVxF domain at the Nterminal end of Rif1 homologues of fungi is present at the C-terminal end of multi-cellular eukaryotes (Figure 2). Thus there has been a swapping of SILK motif in Rif1 from $\mathrm{N}$-terminal end to C-terminal end during the course of evolution. This shift is seen from placozoans onwards, which are the basal group of multi-cellular organisms (Additional file 7). Additionally, in single cell organisms, when the SILK motif is seen in the N-terminus its architecture is 'SILK-RVxF'; but in multi cellular organisms the motif is shifted to C-terminus and the architecture is reversed to
'RVxF-SILK'(Figure 2). Based on the architecture and position of the SILK domain, we again find that the Drosophila homologue is closer to vertebrates than yeasts (Additional file 7). Further analysis of other proteins carrying SILK/PP1 interaction domain in human, yeast and Drosophila showed that the internal swapping of the motifs giving the two

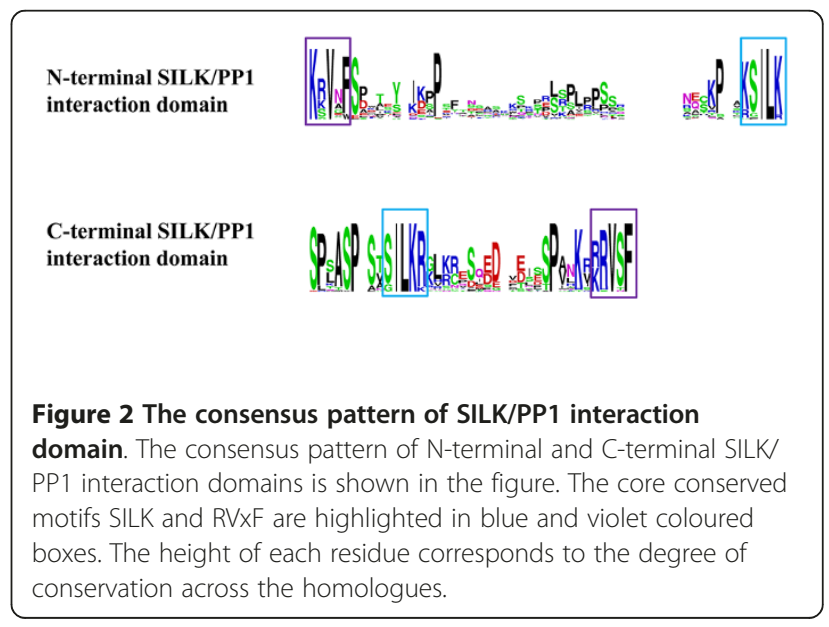


architectures of this domain is not unique to Rif1 (Additional files 8, 9 and 10).

A unique DNA binding domain was reported in hRif1 which helps in bringing the BLM helicase to the stalled replication forks [20]. We found that this domain is conserved from yeast to human (Additional file 11). Although the sequence homology of Rif1 is poor between unicellular and multicellular organisms, the profile based search strongly supports the conservation of this DNA binding domain between these two groups of organisms. BLM interaction domain was also reported in the study of hRif1 by Xu et al. [20]. Our analysis shows that this domain is conserved only in vertebrates (Additional file 12).

In summary, our bioinformatic analyses identified several interesting features of Rif1. We report for the first time the conservation of SILK-RVxF motif in Rif1 from all organisms. We also identify a Rif1 specific core HEAT repeat present in all organisms. The conservation of features of the putative DNA binding domain across species again emphasizes the evolution of the protein from the core sequence and it is important to test if the DNA binding function is also retained.

\section{dRif1 is localized to the nucleus and is prominently associated with heterochromatin}

In order to functionally characterize dRif1, we raised polyclonal antibodies against a part of the protein. The antibody recognized a protein of approximately $160 \mathrm{kDa}$, as expected, in Drosophila embryo derived S2 cell extract (Figure 3A). We performed immunolocalization of dRif1 in S2 cells to see the subcellular localization, and found that Rif1 was nuclear localized (Figure 3B). dRif1 stained the nucleus in a heterogenous manner, with most nuclei showing one or two prominent dark patches along with a diffuse nuclear staining. As the same regions also appeared to contain dense DNA staining, we tested if this patch corresponded to heterochromatin. We colocalized dRif1 with the heterochromatin marker, histone H3 trimethyl lysine 9. As shown in Figure 3C, we found that dRif1 associates with heterochromatin prominently in S2 cells.

\section{dRif1 does not relocalize upon DNA damage induction in S2 cells}

Immunolocalization of human Rif1 shows a diffuse nuclear staining. Multiple forms of DNA damage, including ionising radiation, hydroxy urea, MMS, etoposide, aphidicolin cause hRif1 to relocalize into foci, which often coincide with the damage sites [16-19]. To test if dRif1 also responds to damaged DNA in a similar manner, we treated S2 cells with hydroxy urea and aphidicolin and asked if dRif1 relocalized to halted replication forks. Cells were costained with $\gamma-\mathrm{H} 2 \mathrm{AvD}$ antibodies to mark the sites of damaged DNA. In contrast to what has been observed in human cells, we did not see any major relocalization of dRif1 with either hydoxy urea or aphidicolin treatment (Figure 4A). DNA damage foci that showed strong $\gamma-\mathrm{H} 2 \mathrm{AvD}$ staining were prominent in the treated cells showing that treatment did induce DNA damage. Same results were obtained with MMS and UV treatments (data not shown). Therefore, dRif1, unlike hRif1, does not relocalize upon DNA damage.
A

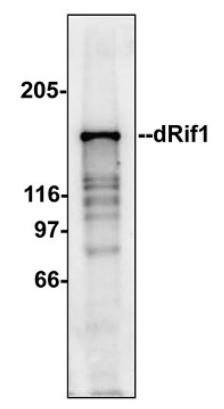

C

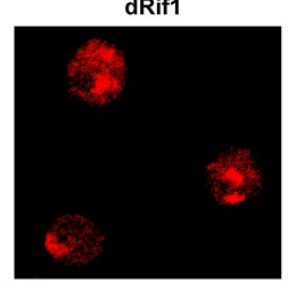

B
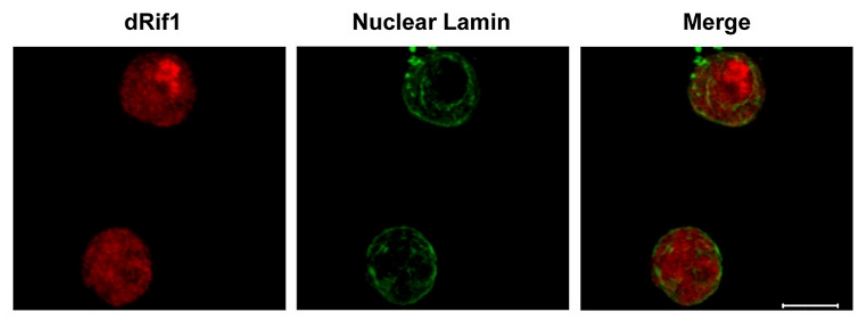

$\mathrm{H}_{3 \mathrm{~K} 9 \mathrm{Me}_{3}}$

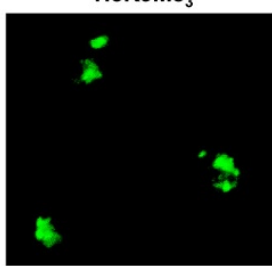

TO-PRO3

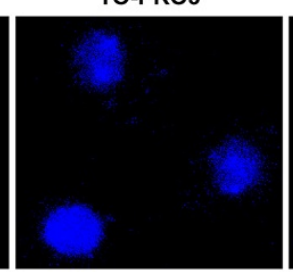

Merge

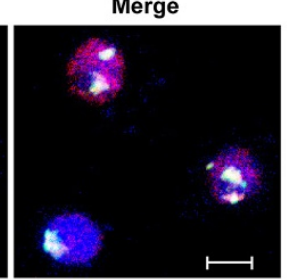

Figure 3 dRif1 localizes to the nucleus in unperturbed cells. A) Western blot of total protein extract from S2 cells was probed with antibody against dRif1. A $160 \mathrm{kDa}$ lights up prominently. B) S2 cells were immunostained with antibodies to dRif1 (red) and costained with antibodies to lamin (green) to mark the nucleus. C) S2 cells were immunostained with antibodies to H3K9 trimethyl (green) and dRif1 (red) and TO-PRO3 to mark the nucleus (scale bar, $5 \mu \mathrm{m}$ ). 


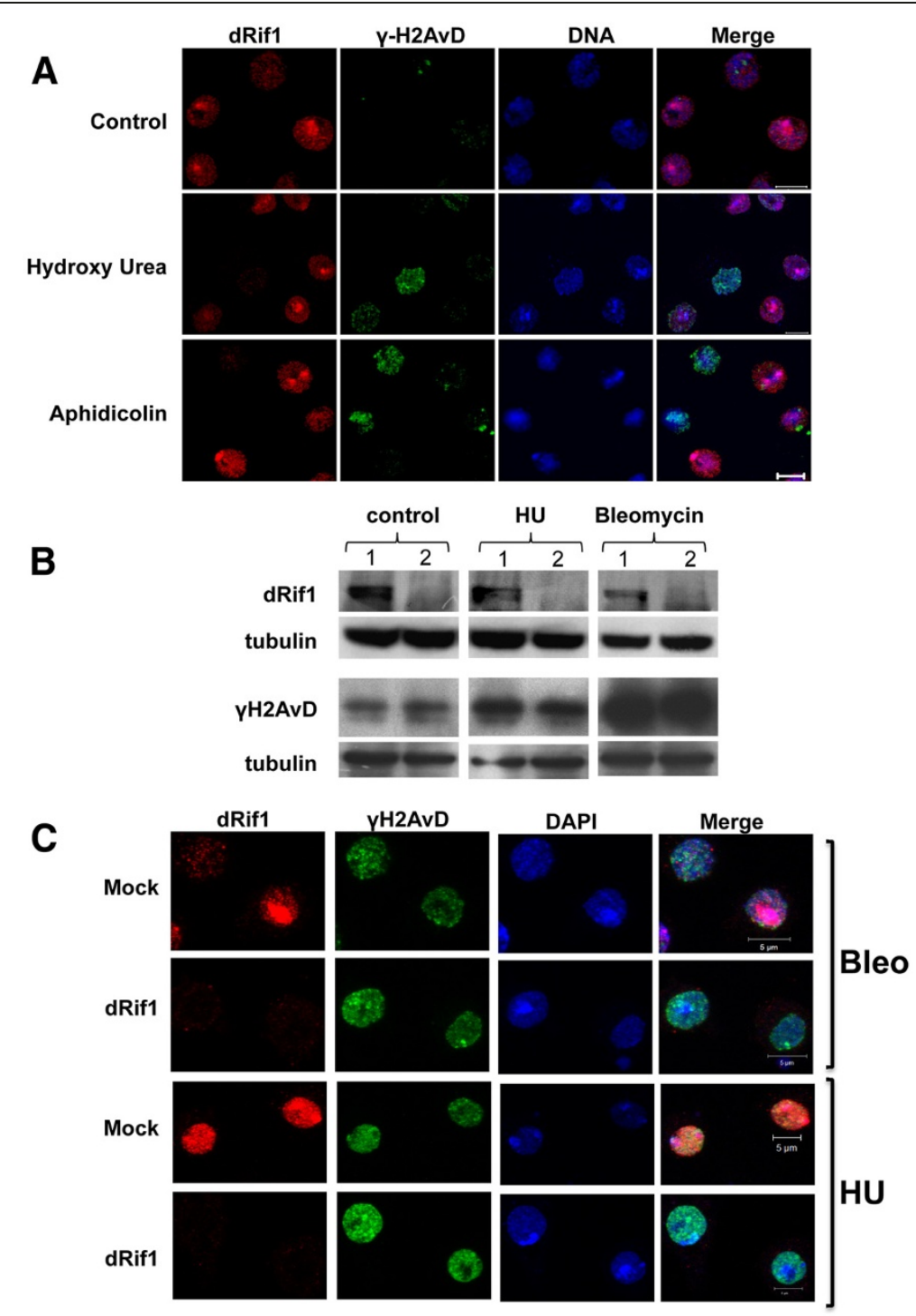

Figure 4 dRif1 does not colocalize to the DNA damage foci induced by $\mathrm{HU}$ and Aphidicolin. A) S2 cells were treated with either $2.5 \mathrm{mM}$ hydroxy Urea (HU) or $25 \mu \mathrm{M}$ aphidicolin for $16 \mathrm{hrs}$ to induce DNA damage and then fixed, stained with antibodies to dRif1 (red) and $\mathrm{HH} 2 \mathrm{AvD}$ (green). The slides were mounted in mounting media containing DAPI or TO-PRO 3 (blue). B) S2 cells were treated with either dsRNA of GFP (1) or dRif1 (2). Each was further split into three parts and was either mock treated or treated with hydroxyurea (HU) or bleomycin. Protein extracts were tested for dRif1 and $\gamma H 2 A v D$ expression; tubulin was used as a loading control. dRif1 was undetectable in RNAi treated cells. $\gamma H 2 A v D$ levels increase upon exposure to DNA damage. C) The cells were also immunostained with $\gamma \mathrm{H} 2 \mathrm{AvD}$ to detect DNA damage sites. All the cells were fixed and stained for dRif1 (red), YH2AvD (green) antibody and DAPI (blue) Scale bar is $5 \mu \mathrm{m}$.

The experiments described above showed that dRif1 does not respond to DNA damage by localizing to the repair sites. However, in order to test this more directly, we carried out knock down experiments using doublestranded RNA. Three different primer sets with no off targets were designed for dRif1. We used dsRNA of GFP for the mock treatment experiments. We did two successive rounds of dsRNA treatment and performed both western blot and immunofluorescence studies and confirmed that dRif1 protein levels decreased to undetectable levels by the sixth day (Figure 4B). Cells remained healthy and continued to divide for several days after dsRNA treatment. These knockdown cells were treated with DNA damage inducing agents, $\mathrm{HU}$ and bleomycin. After treatment we stained the cells for $\gamma-\mathrm{H} 2 \mathrm{AvD}$ and dRif1. First, we did not find any difference in viability between mock treated and double stranded dRif1 RNA treated cells upon induction of DNA damage. Second, upon staining for $\gamma$-H2AvD, we found several spots come up on DNA damage induction (Figure 4C). We compared the levels of $\gamma-\mathrm{H} 2 \mathrm{AvD}$ between wild type and knock down cells by western blots (Figure 4B). Our 
results show, both by immunofluorescence and by western blots, that repair foci (therefore, signaling of DNA damage) occur normally in the absence of dRif1. These data further strengthen our conclusions that unlike human Rif1, Drosophila Rif1 does not participate in DNA damage response by translocating to the sites of repair.

\section{Knock down of dRif1 does not influence telomere transcription in S2 cells}

As yRif1 represses transcription of telomeric repeat containing transcripts (TERRA) [27,28] and is involved in telomere position effect in C. glabrata, we asked if telomere specific transcript levels are under dRif1 control in Drosophila. Drosophila telomeres have retrotransposon elements that are generally transcriptionally suppressed and are activated by mutations that inactivate telomere position effect and rasiRNA pathway, indicating that they are under strict transcriptional repression (reviewed in [29]). We isolated RNA from wild type and knock down cells and performed quantitative reverse transcriptase PCR for transcripts from telomere associated transposons [30].We do find low levels of transcripts in wild type cells and this level is not significantly affected by knock down of Rif1 (Figure 5). This result suggests that dRif1 is unlikely to regulate telomere retrotransposon transcript levels in S2 cells.

\section{dRif1 does not complement telomere function in yeast}

The bioinformatic analyses presented above show that Rif1 is conserved throughout eukaryotes. However, as shown above, we found that dRif1 responded differently to DNA damage in comparison to human Rif1. To test

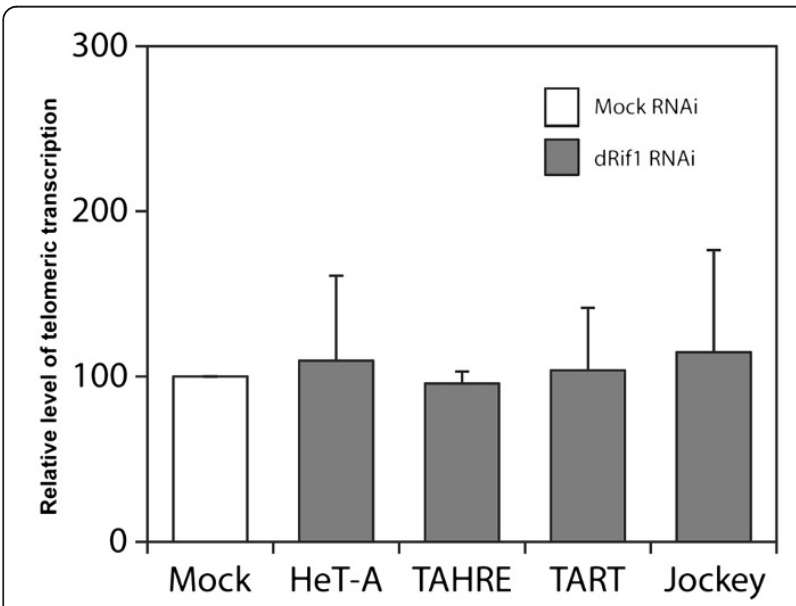

Figure 5 dRif1 knock down does not affect telomeric transcription in S2 cells. RNA was extracted from mock and dRif1 dsRNA treated cells and RT-PCR was performed to detect levels of telomere associated retrotransposon transcripts (HeT-A, TAHRE, TART and jockey, the internally located retrotransposon). how much of the core functional properties of yeast Rif1 are retained in Drosophila, we performed cross complementation assays. To this end the full length dRif1 was expressed under the control of yeast Rif1 promoter and transformed into wild type, rif1, rif2 and rif1rif2 mutant yeast strains and the telomere length was estimated. Yeast lacking Rif1p have much longer telomeres than wild type cells $[5,6]$. As seen in Figure 6, wild type cells have compact telomeres around $1.2 \mathrm{~kb}$ (lanes 1, 3\&11) rif1 (lanes $6 \& 8$ ) and rif2 (lanes 12\&14) mutations increase the length of the telomeres considerably, with rif1 mutants showing more pronounced effects. The double mutants have an additive effect and the telomeres are extremely long and disperse (lanes 17\&19). Single copy expression of yeast Rif1 is able to complement rif1 phenotype (lanes 5, 10, 16\&21); however, dRif1 does not change the telomere length in any of these strains (wild type- lanes $2 \& 4$, rif1lanes7\&9, rif2- lanes $13 \& 15$ and rif1rif2- lanes 18\&20). Apart from telomere length, yeast Rif1 has a negative regulation on telomere position effect (TPE). Therefore, we tested whether TPE in yeast is perturbed by dRif1 expression. However, dRif1 expression does not affect TPE either (Additional file 13). This suggests that the Drosophila protein does not retain much functional similarity to its yeast counterpart and therefore, cannot complement yRif1 in telomere maintenance. This is in contrast to hRif1, which increases telomere length in rif2 mutants [17]. These data lead us to speculate that unlike hRif1, Drosophila Rif1, has lost the ability to interact with and interfere with telomere length regulation in yeast.

As a further test of functional conservation of dRif1, we determined the subcellular localization of dRif1 when expressed in yeast. We first confirmed by western blot analysis that dRif1 was expressed in yeast cells. As seen in Figure 7A, FLAG-tagged Rif1 could be detected in yeast. Empty vector or FLAG-tagged dRif1 was transformed into yeast strains that express myc-tagged Sir4 protein. Sir4, silent information regulator 4 protein, localizes to telomeric clusters and appears as 3 to 6 bright foci in the nuclei. These strains were fixed and immunofluorescence experiments were performed using FLAG (dRif1) and myc (Sir4) antibodies. We found that 20-30 percent of the cells have clear nuclear signal for dRif1, showing that dRif1 localizes to the nucleus in yeast (Figure 7B; panels 2 and 4). Empty vector transformed control cells did not show any signal for dRif1. However, there was no colocalization of the dRif1 with Sir4 protein. Therefore we conclude that dRif1 protein does not localize to the telomeres in yeast and this also explains the failure of dRif1 in complementing its yeast counterpart in our previous experiments. Since hRif1 has been shown to localize to aberrant, unprotected human telomeres, we tested dRif1 localization in $y k u 70$ mutants as $y k u 70$ mutant have damaged telomeres; but no relocalization of dRif1 to these sites could be detected 


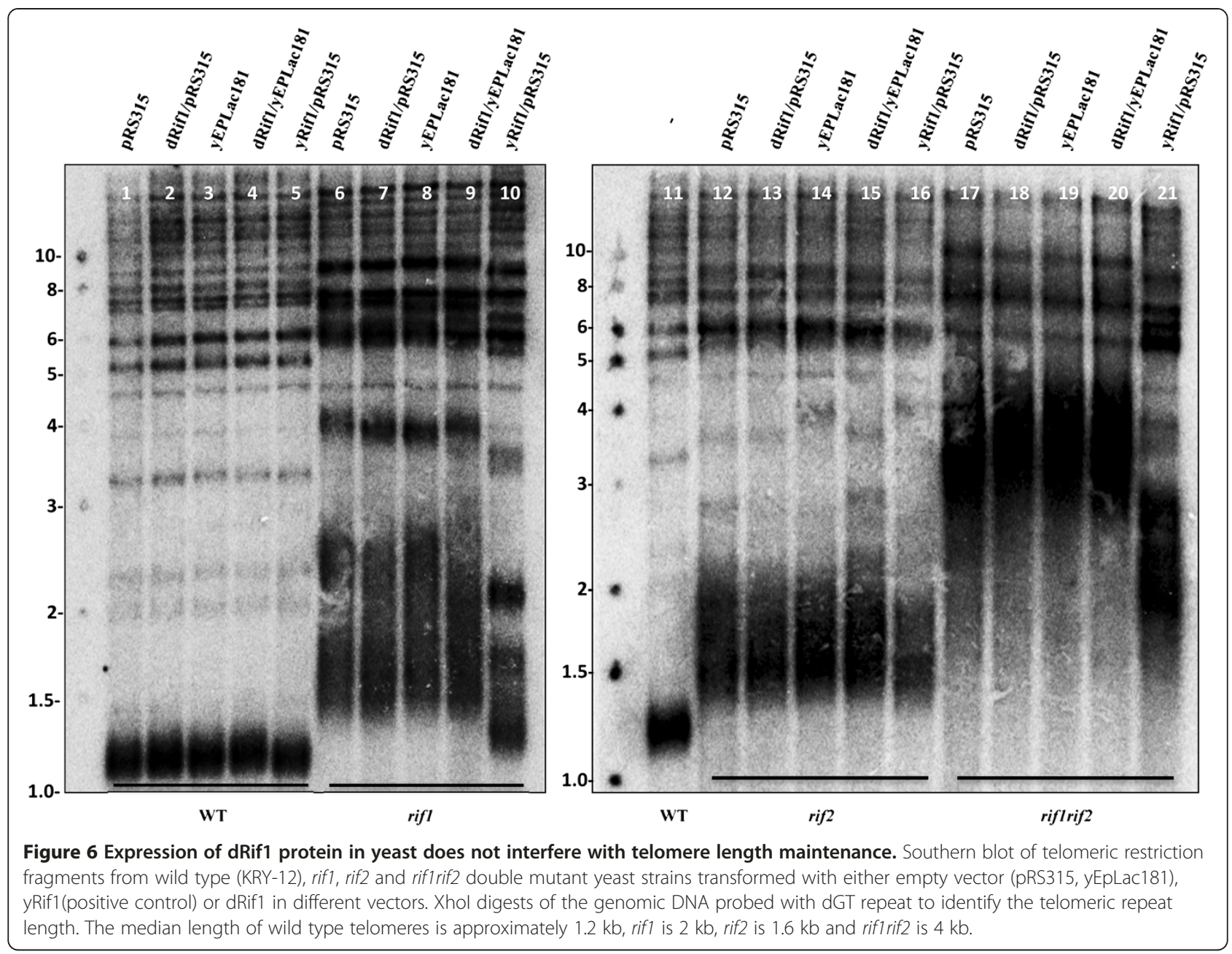

(data not shown). There were two interesting features about dRif1 localization in yeast. First, only a subset seemed to show dRif1 staining, suggesting that not all cells were expressing dRif1. Second, dRif1 localized to a distinct compartment within the nucleus, which is neither telomeres, nor nucleolus. This novel site usually appeared as bright spot in the nucleus and sometimes appeared as a ring. Such novel site localization has recently been reported for Slx5, a component of the ubiqutin E3 ligase complex that targets sumoylated proteins and reported to have roles in DNA damage response [31]

Mammalian Rif1 is localized to the nucleus and relocalizes to the DNA damage/repair foci $[16,17]$. We induced DNA damage in yeast cells expressing dRif1 by incubating the overnight grown cultures with $0.05 \% \mathrm{MMS}$ and checked for the dRif1 localization pattern upon DNA damage (Figure 7B, panels 3 and 4). We found that the staining remained the same and Rif1 retained its unique pattern; although now most of the nuclei showed the more prominent ring like localization unlike the prominent spot or small ring staining in the untreated cell nuclei. As reported, the Sir4p spots became more diffuse [32,33]. These data suggest that dRif1 does not relocalize to DNA damage sites in yeast as well.

\section{dRif1 does not co-localize with DNA damage sites in human cells}

The lack of relocalization of dRif1 to sites of damage in yeast could be either due to lack of conservation of the partners or pathways in yeast or alternatively might indicate lack of conservation of this function in dRif1. In order to distinguish between the two possibilities, we expressed FLAG-dRif1 in HeLa cells. The full-length dRif1 along with 3XFLAG was cloned in PCMV vector and transfected into HeLa cells. Since dRIF1 colocalized with heterochromatin in S2 cells, we stained dRif1 using FLAG antibody to confirm transfection and also co-stained with H3K9Me3 antibody as heterochromatin marker. We did not find any significant colocalization of dRif1 with heterochromatin in HeLa cells (Additional file 14). After 24 hrs of transfection, the cells were treated with $\mathrm{HU}$ for $16 \mathrm{hrs}$ or bleomycin for 4 hours and both treated and untreated cells were stained with antibodies to 53BP1 and FLAG. Untreated control 


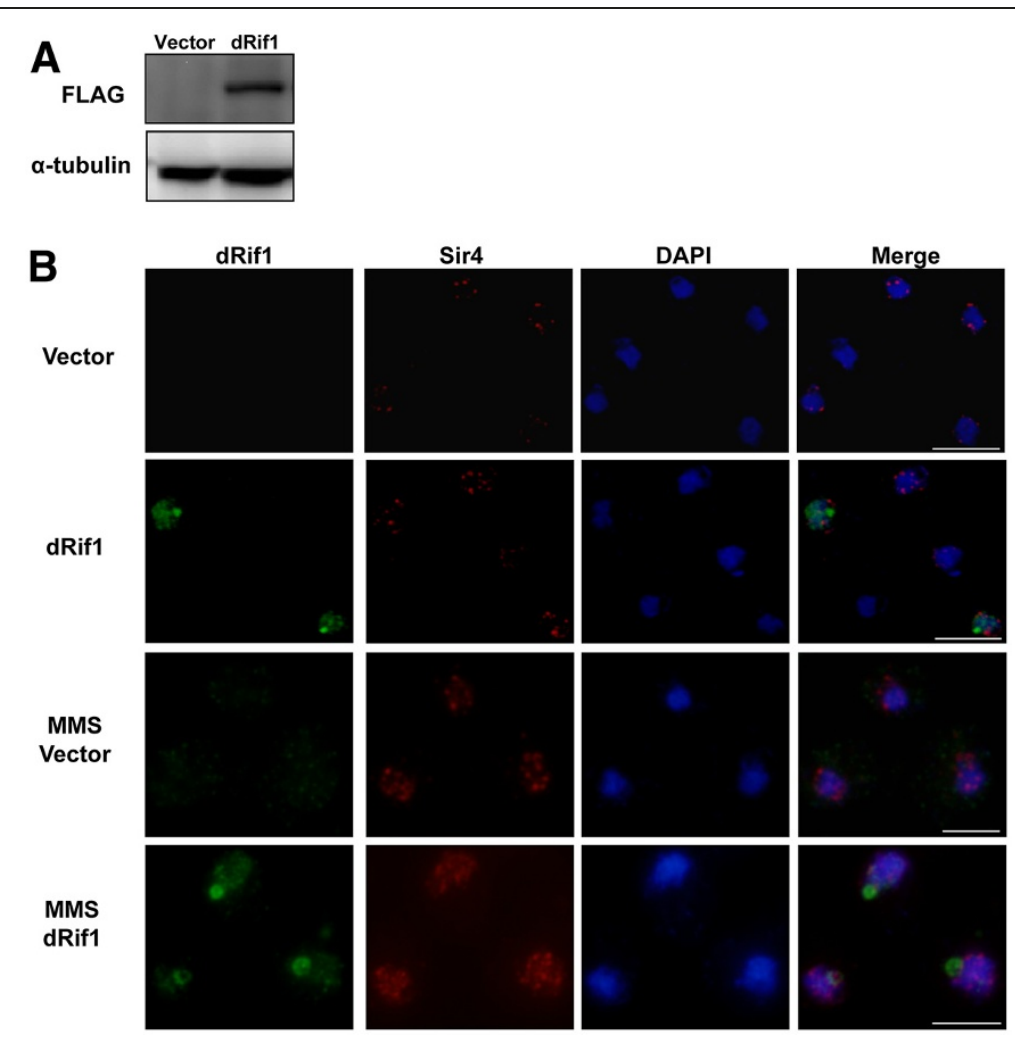

Figure 7 A) FLAG tagged dRif1 is expressed in S.cerevisiae. Total cell extracts of yeast transformed with empty vector (lane 1) or 3XFLAG tagged dRif1 (lane 2) were probed with antibodies to FLAG and tubulin. Lanes 2 shows 3XFLAG dRif1 expression. B) dRif1 introduced in yeast localizes to the nucleus but not to yeast telomeres. Yeast cells transformed with empty vector or 3X FLAG dRif1 were stained for 3xFLAGdRif1 (green) and 13xmycSir4 (red). Nuclei were stained with DAPI (blue; Panel B, row 1\&2). DNA damage induction with 0.05\%MMS for 90 minutes treatment did not alter the localization of dRif1 in yeast (Panel B, rows 3,4). (Scale bar, 5 Hm).

cells showed clear nuclear localization of FLAG tagged dRif1 where as 53BP1 showed one or two foci (Figure 8, row1 and 3).

When HeLa cells were treated with HU (row2) or bleomycin (row4), we found that damage sites were marked with 53BP1. Eventhough hRif1 has been shown to accumulate at such damage foci, we observed that dRif1 did not accumulate in these sites [16-18]. An interesting feature of expressing dRif1 protein in HeLa cells was that when a larger amount of protein was expressed, the nuclei appeared deformed and additionally, did not show large 53BP1 spots or foci upon HU or bleomycin treatment. However, in cells not expressing dRif1, or expressing low levels of dRif1, prominent 53BP1 spots were observed, even though dRif1 did not colocalize with these damage spots, indirectly suggesting that dRif1 protein possibly interfered with the normal DNA damage response of HeLa cells. These data along with our previous result showing that dRif1 does not accumulate at DNA damage sites in Drosophila cells as well, suggest that the Drosophila homologue may not respond to DNA damage in the same manner as the human homologue.

\section{Discussion}

Rif1 was identified in yeast almost two decades ago, and genetic and biochemical studies have clearly shown that it is a negative regulator of telomerase. Emerging evidence shows that Rif1 in mammals has diverged from its primary role in telomere synthesis and maintenance to a broader role in response to DNA damage. In this work we initiated a study on the Drosophila homologue of Rif1. Our detailed analysis of Rif1 from multiple organisms has identified several novel features. Drosophila Rif1 is evolutionarily closer to vertebrate Rif1 than yeast Rif1. All Rif1 homologues contain the conserved HEATrepeats and this may carry out the core Rif1 activities. As this domain has been implicated in interacting with proteins, it might recruit a variety of proteins to carry out its functions. Within these HEAT repeats, our studies identify a conserved Rif1 specific repeat and this might be useful in identifying the core conserved interacting partners. The more diverse repeats are likely to facilitate participation in other functions. Our analysis also identified a conserved SILK motif, again present in all organisms, from yeast to humans. As this motif has been retained in all species, this is likely to participate in 


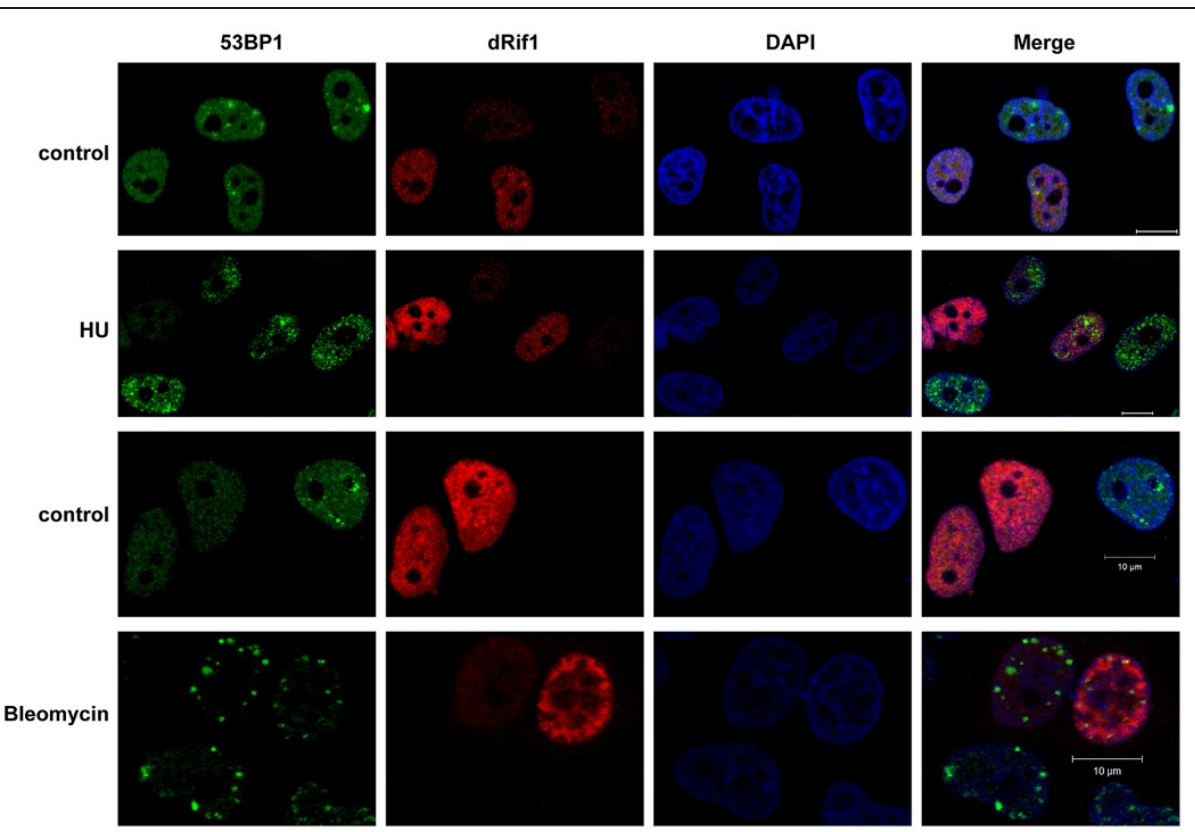

Figure 8 Heterologous-expression of dRif1 in HeLa cells interferes with the 53BP1- foci formation upon DNA damage. HeLa cells were transfected with full length dRif1 with a N-terminal 3XFLAG tag. Cells were either mock treated (row 1,3) or with $2 \mathrm{mM} \mathrm{HU}$ (row 2) or $50 \mu \mathrm{gg} / \mathrm{ml}$ of bleomycin (row 4) for $4 \mathrm{hrs}$, fixed and stained for dRif1(red) and 53BP1 (green). DAPI (blue) was used to mark the nuclei. (Scale bar, $10 \mu \mathrm{m}$ ).

the core Rif1 functions. dRif1 lacks the C-terminal BLM interaction domain but contains all the conserved features associated with the $\mathrm{N}$-terminal region.

$\mathrm{dRif1}$ encodes for a $160 \mathrm{kDa}$ protein that is localized to the nucleus. We find that a large fraction of the protein is associated with heterochromatin. In budding yeasts, yRif1 is predominantly associated with telomeric heterochromatin, although it is not required for establishment or maintenance of telomeric heterochromatin [9]. A very recent report implicates yRif1 in heterochromatin establishment at the silent mating type loci [34] and genome wide chromatin immunoprecipitation studies also show that yRif1 is associated with the silent mating type loci [11]. Rif1 in human cell lines were also shown to be associated with arrested replication forks in the vicinity of pericentromeric heterochromatin although this was not observed in unperturbed cells [18]. These results taken together implicate a possible evolutionarily conserved role for Rif1 at the heterochromatin.

We find that knock down of dRif1 does not lead to any difference in response to DNA damage in S2 cells suggesting that dRif1 is unlikely to function at repair sites. Drosophila and human Rif1 behave differently in yeast: whereas human Rif1 interferes with telomere length in yeast [17], Drosophila Rif1 does not. This suggests that human Rif1 has possibly retained its ability to interact with telomeric partners of Rif1, possibly Rap1, and Drosophila Rif1 has lost that ability perhaps, because unlike yeasts and vertebrates, Drosophila does not have Rap1. In this context, it is important to note that out of the 325 genomic targets identified for Rif1 in yeast, only about 88 , mostly telomeric, colocalize with Rap1, suggesting there are a large number of Rap1 independent targets for Rif1 even in yeast [11]. We speculate that telomere independent functions of Rif1 are conserved in Drosophila and yeast and need to be explored.

Interestingly, upon DNA damage, dRif1 does not associate with the DNA repair foci although human Rif1 does. In fact, presence of dRif1 reduces the formation of DNA repair foci in HeLa cells. The C-terminus of vertebrate Rif1 has now been shown to interact with the BLM complex and also contain a DNA binding domain [20]. However, the Drosophila homologue does not have the extended C-terminus that carries out the critical functions of association with BLM protein. This suggests that Drosophila Rif1 may not have the ability to associate with replication forks and the differential response of dRif1 and human Rif1 to the presence of stalled replication forks are consistent with this.

The retention of Rif1 homologue in Drosophila raises an important question as to when and how Rif1 function diversified. As telomerase based telomere maintenance was replaced by alternate mechanisms of maintenance in many insects including Drosophila, telomerase and associated proteins have no counterparts in these organisms [35]. However, presence of Rif1 in Drosophila suggests that the recruitment of Rif1 to non-telomere based roles happened before Drosophila lost telomerase. Alternatively it might mean that Rif1 has both a telomeric and an evolutionarily conserved non-telomeric role in yeast. 
Even in yeast, only the C-terminus of Rif1 has been shown to interact with Rap1 and Rif2 proteins. The conserved N-terminal domain containing HEAT repeats has so far not been implicated in any function. Could this domain hold the clue to the evolutionarily conserved role of Rif1? Even though Rif1 was found as a negative regulator of telomerase, it has now been implicated in many more (previously unanticipated) functions like in telomere protection, recombination mediated telomere maintenance and repression of telomere specific transcripts $[7,28,36]$. However a molecular or biochemical basis underlying these functions is lacking. No specific motifs have been identified in Rif1 that could predict a biochemical function. Indeed there has been no structure -function analyses performed for any of the diverse Rif1 functions in yeast. Comparing the sequences of Rif1 throughout eukaryotes and experimental data obtained for Rif1 from the various model systems, it appears that the core conserved region of Rif1, the HEAT repeats and SILK motifs, warrant special attention. Studies in a genetically and developmentally tractable system like Drosophila will give us an additional important handle to understand the function of this conserved protein.

\section{Conclusion}

In this study, we have carried out a detailed bioinformatic analysis of Rif1 and show that it is evolutionarily conserved across eukaryotes. Our study shows that within the HEAT repeats, there is a core Rif1 specific repeat region that is present in all the Rif1 homologues. A PP1 docking motif has also been identified in all Rif1 homologues. dRif1 is localized to the nucleus and shows a prominent heterochromatin association. It does not localize to foci induced by DNA damage. When tested for functional conservation of Rif1 function in dRif1, we find that it does not perturb or complement yeast Rif1 and does not relocalize to DNA damage foci in HeLa cells. The novel motifs identified in this study give a new perspective to investigate Rif1 functions, especially with respect to PP1 interactions and heterochromatin associations. Secondly, whether responding to DNA damage or binding to stalled replications forks is a newly acquired vertebrate specific function of Rif1 needs to be examined. This would suggest a further evolution and sub-functionalization of an ancient protein.

\section{Methods}

\section{Bioinformatics methods}

Rif1 protein sequence of human and yeast were used for finding the homologues in NCBI protein sequence database using PSI BLAST [37]. After three rounds of iteration, irrespective of their percentage of sequence homology, all the sequences were considered as putative homologues and subjected to motif prediction by MEME tool [38]. The motifs which are common between human and yeast Rif1 were considered as conserved domains across the homologues. HMM profiles were generated for the motifs using HMMer tool and was used to mine the NCBI protein sequence database [39]. This reverse profile based search strategy was helpful for us to mine the true homologue. The multiple sequence alignment of protein and motif sequences was done using ClustalW and ClustalX [40,41]. The Rif1 homologues were subjected for phylogenetic analysis using ClustalX neighbour joining algorithm [40]. The tree was constructed with 1000 replicates with a random bootstrap value. The consensus tree was visualized using MEGA software [42]. A simple pattern search program was written in Perl to identify the core conserved residues of PP1 interaction domain/SILK motifs and the search was done in the NCBI protein sequence build for human, Drosophila and yeast.

\section{Cloning and expression of dRif1}

Full-length cDNA clone of CG30085-dRif1 (RE66338) was obtained from the Drosophila Genomics Resource Center (DGRC). In order to tag the dRif1 protein, we designed an oligonucleotide encoding 3X FLAG tag with sequence for NcoI compatible over hang at both the ends, annealed to get double stranded DNA, and cloned in frame at the start of the dRif1 in RE66338. The sequence and orientation of the tag was confirmed by DNA sequencing. To express the protein in yeast, we inserted the full length 3xFLAG tagged construct in pRS315 and yEpLac-181 vectors under the control of the yeast Rif1 promoter. These plasmids were transformed into yeast strain (KRY-109) containing a Sir413xMyc. The same 3xFLAG tagged full length protein was transferred to pCMV vector for HeLa cell experiments.

\section{Yeast transformation and telomere blots}

Yeast transformation was done using lithium acetate mediated transformation and DNA was isolated using zymolyase. Genomic DNA was isolated from all the strains (wild type, rif1, rif2 and rif1rif2 mutants expressing empty vector, yRif1 or dRif1). Approximately $1.5 \mu \mathrm{g}$ of genomic DNA was digested with Xhol and subjected to electrophoresis on a $0.8 \%$ agarose gel along with $1 \mathrm{~kb}$ ladder (New England Biolabs). The gel was soaked in $0.4 \mathrm{~N} \mathrm{NaOH}$ for $10 \mathrm{~min}$, and capillary transferred to charged Nylon membrane (IMMOBILON-NY+, Millipore) using 0.4 N NaOH. The membrane was hybridized to the radiolabelled dGT/ $\mathrm{CA}$ repeat at $55^{\circ} \mathrm{C}$. XhoI cuts yeast genomic DNA at a conserved site that is $1.5 \mathrm{~kb}$ upstream of the telomere repeats and when probed with telomere sequence, the size of this band indicates the length of the telomere.

\section{Immunofluorescence}

Yeast cells were grown in SC-Leu selective media overnight and the cells were fixed with formaldehyde, spheroplasted 
and spotted on a poly-lysine coated multi-well slide. It was then treated with pre-chilled methanol and acetone for further permeabilization. The cells were then blocked in PBST (0.1\% triton X100) containing $0.1 \%$ BSA and incubated with appropriately diluted primary antibodies in PBST overnight at $4^{\circ} \mathrm{C}$. Washes were performed to remove unbound antibodies and incubated with secondary antibodies conjugated with fluorophores, washed again and mounted with mounting media containing DAPI. In yeast, DNA damage was induced by treating the cells with $0.05 \%$ of MMS (SIGMA) for 90minutes. After that the cells were harvested and immunofluorescence performed as described before and the images were captured in an Olympus IX81 microscope. For HeLa and S2 cells, cells were plated and grown on cover slips. They were formaldehyde fixed, permeabilized, blocked and stained using antibodies indicated at appropriate dilutions. The slides were imaged in a multiphoton LSM-510 Zeiss confocal or LSM-710 Zeiss confocal microscope. Antibodies used in the study are H2AvD pS137 (Rockland Immunochemicals), 53BP1 (Santacruz), Myc (Abcam) and FLAG-M2 (Sigma). Polyclonal antibodies to dRif1 were raised in rabbit. Amino acids 694-1094 of dRif1 was expressed in bacteria as a $6 \mathrm{x}-\mathrm{HIS}$ tagged fusion protein; purified and immunized a rabbit for antibody production. The serum was affinity purified against the same bacterially expressed protein bound to nitrocellulose membrane before use.

\section{Knock down of dRif1 in S2 cells}

Double stranded RNA (dsRNA) was used to knockdown dRif1 levels in S2 cells. Three different primer sets were designed (with no off target) along with 5' T7 binding site. Primer sequences will be provided upon request. GFP dsRNA was used as control/mock experiment. MEGAscript T7 kit from Ambion was used to make dsRNA according to the manufacturer's instructions and checked on gel for integrity of RNA made and stored at $-20^{\circ} \mathrm{C}$ until use. $1 \times 10^{6}$ cells $/ \mathrm{ml}$ of S2 cells were treated with $\sim 30 \mu \mathrm{g}$ of the dsRNA in serum free media for 30 minutes and later supplemented with serum containing media. After 4 days one more round of dsRNA treatment was given to completely knockdown dRif1. These cells were then processed for RNA isolation, IF and DNA damage induction treatment. 2.5 mM HU for $16 \mathrm{hrs}, 50 \mu \mathrm{g} / \mathrm{ml}$ bleomycin for $4 \mathrm{hrs}$ were used for the DNA damage induction. Cells were harvested and total protein and RNA were made from untreated and treated samples. A fraction of the same sets of cells were processed for immunofluorescence.

\section{RNA isolation and RT PCR}

RNA isolation was carried out using trizol method and treated with RNase free DNase. $2 \mu \mathrm{g}$ of RNA was used to make cDNA and to check the telomeric transcription levels. Telomeric transcription was assessed by comparative $\mathrm{C}_{\mathrm{T}}$ method in ABI 7500FAST machine using SYBER GREEN chemistry using primers mentioned below $[30,43]$. Signals were normalized against Rps17 as internal control. The telomeric transcript levels of dRif1 RNA treated samples were compared against GFP RNA treated samples (set as 100). Graphs were plotted with relative level of telomeric transcription for each locus. Averages are from two independent experiments and error bars indicate standard deviation. Sequence of primers used to detect the telomeric and control transcripts are mentioned below.

$\mathrm{RpS} 17-\mathrm{F} \rightarrow$ AAGCGCATCTGCGAGGAG
$\mathrm{RpS} 17-\mathrm{R} \rightarrow$ CCTCCTCCTGCAACTTGATG
$\mathrm{HeT}-\mathrm{F} \rightarrow \mathrm{TTGTCTTCTCCTCCGTCCACC}$
$\mathrm{HeT-R} \rightarrow$ GAGCTGAGATTTTCTCTATGCTACTG
$\mathrm{TAHRE}-\mathrm{F} \rightarrow$ CTTCCCCTCCGCTCTCATC
TAHARE-R $\rightarrow$
CCTAGATCTGCATTTGTATTAGTAGCTG
TART-F $\rightarrow$ CAAAAAATCCTTTCCGAGATCC
TART-R $\rightarrow$ GGGCATCAATATTTAGAATGAACAG
Jockey-F $\rightarrow$ ACGACTCAATCTAGGGCTCGTG
Jockey-R $\rightarrow$ CGTCCATTCTCGTATTGATGG

\section{Additional files}

Additional file 1: The list of Rif1 homologues. The organism name common name and the NCBI accession number of the Rif1 homologues are given in the table.

Additional file 2: Expanded Phylogenetic tree of Rif1 homologues. The consensus phylogenetic tree of Rif1 homologues drawn by the neighbour joining method is shown. The random sampling was done for 1000 replicates and the branches having bootstrap value above 50 percentage are shown in the figure. The corresponding protein sequence accession number for the organisms mentioned in the tree is given the Additional file 1

Additional file 3: Putative plant homologues of Rif1. The organism name, common name and the NCBI accession number of the Rif1 homologues are given in the table.

Additional file 4: The core conserved region of HEAT repeat. The organism name and the length of the domain for each sequence are shown to the left and right of the multiple sequence alignment, respectively. The amino acids are highlighted in different colours based on their property. The degree of conservation at each position in the alignment is represented as bar graph at the bottom of the alignment.

Additional file 5: The N-terminal SILK/PP1 interaction domain of unicellular organisms. The organism name and the length of the domain for each sequence are shown to the left and right of the multiple sequence alignment, respectively. The amino acids are highlighted in different colours based on their property. The degree of conservation at each position in the alignment is represented as bar graph at the bottom of the alignment.

Additional file 6: The C-terminal SILK/PP1 interaction domain of multicellular organisms. The organism name and the length of the domain for each sequence are shown to the left and right of the multiple sequence alignment, respectively. The amino acids are highlighted in different colors based on their property. The degree of conservation at each position in the alignment is represented as bar graph at the bottom of the alignment. 
Additional file 7: The domain shift relationship among organisms that have Rif1. The organisms having N-terminal or C-terminal SILK/PP interaction domain are highlighted in red and green, respectively in the tree.

\section{Additional file 8: The list of proteins with SILK/PP1 interaction} domain in Homo sapiens. The NCBI accession number, protein name, SILK/PP1 interaction domain, protein size, domain length and the position of the motif for the proteins having SILK/PP1 interaction domain are listed in the table.

Additional file 9: The list of proteins with SILK/PP1 interaction domain in Drosophila melanogaster. The NCBI accession number, protein name, SILK/PP1 interaction domain, protein size, domain length and the position of the motif for the proteins with SILK/PP1 interaction domain are listed in the table.

Additional file 10: The list of proteins with SILK/PP1 interaction domain in Saccharomyces cerevisiae. The NCBI accession number, protein name, SILK/PP1 interaction domain, protein size, domain length and the position of the motif for the proteins with SILK/PP1 interaction domain are listed in the table.

\section{Additional file 11: The DNA binding domain of hRif1 is conserved} across the homologues. The organism name and the length of the domain for each sequence are shown to the left and right of the multiple sequence alignment, respectively. The amino acids are highlighted in different colours based on their property. The degree of conservation at each position in the alignment is represented as bar graph at the bottom of the alignment.

Additional file 12: The BLM1 interaction domain of hRif1 is conserved across vertebrates. The organism name and the length of the domain for each sequence are shown to the left and right of the multiple sequence alignment, respectively. The amino acids are highlighted in different colours based on their property. The degree of conservation at each position in the alignment is represented as bar graph at the bottom of the alignment

Additional file 13: Telomere position effect is not altered by expression of dRif1 in yeast. Wild type, rif1, rif2 and rif1rif2 strains were transformed with empty vectors (pRS315, yEPLac181), dRif1 (pRS315dRif1, yEPLac181dRif1) and yeast Rif1 (pRS315yRif1). All strains contain URA3 gene at the telomere of chromosome VIIL. yku70 mutant is a positive control for loss of gene silencing; URA3 is expressed and therefore not growing on FOA plate. The silencing on FOA plates with tenfold dilution and spotting assay do not show difference in growth compared to the corresponding vector alone control in wild type, rif1, rif2 and rif1rif2 double mutant strains.

Additional file 14: dRif1 does not significantly colocalize with $\mathrm{H}_{3} \mathrm{~K}_{\mathrm{MMe}}$ in HeLa cells. HeLa cell transfected with FLAG-dRif1 were stained with anti-FLAG antibody (red) and H3K9Me3 antibody (green). DAPI is seen as blue staining.

\section{Competing interests}

The authors declare that they have no competing interests.

\section{Acknowledgements}

KM acknowledges support from CSIR, India, DST-FIST and UGC-SAP; ES and RS acknowledge CSIR for fellowship, RM lab is funded by CSIR.

\section{Author details}

'Department of Biochemistry, School of Life Sciences, University of Hyderabad, Hyderabad 500046, India. ${ }^{2}$ Centre for Cellular and Molecular Biology, Council of Scientific and Industrial Research, Uppal Road, Hyderabad 500 007, India.

\section{Authors' Contributions}

KM conceived the study, KM and RM designed the study, RS performed the bioinformatic analysis, ES performed the experiments, VB performed experiments with flies, KM, ES, RS and RM analysed the data, and KM wrote the paper. All authors read and approved the final manuscript.
Received: 4 November 2011 Accepted: 19 June 2012

Published: 19 June 2012

\section{References}

1. Bianchi A, Negrini S, Shore D: Delivery of yeast telomerase to a DNA break depends on the recruitment functions of $\mathrm{Cdc} 13$ and Est1. Mol Cell 2004, 16(1):139-146.

2. Osterhage JL, Friedman KL: Chromosome end maintenance by telomerase. J Biol Chem 2009, 284(24):16061-16065.

3. O'Sullivan RJ, Karlseder J: Telomeres: protecting chromosomes against genome instability. Nat Rev Mol Cell Biol 2010, 11(3):171-181.

4. Smogorzewska A, de Lange T: Regulation of telomerase by telomeric proteins. Annu Rev Biochem 2004, 73:177-208.

5. Hardy CF, Sussel L, Shore D: A RAP1-interacting protein involved in transcriptional silencing and telomere length regulation. Genes Dev 1992, 6(5):801-814.

6. Wotton D, Shore D: A novel Rap1p-interacting factor, Rif2p, cooperates with Rif1p to regulate telomere length in Saccharomyces cerevisiae. Genes Dev 1997, 11(6):748-760.

7. Hirano Y, Fukunaga K, Sugimoto K: Rif1 and rif2 inhibit localization of tel1 to DNA ends. Mol Cell 2009, 33(3):312-322.

8. Teng SC, Chang J, McCowan B, Zakian VA: Telomerase-independent lengthening of yeast telomeres occurs by an abrupt Rad50p-dependent, Rif-inhibited recombinational process. Mol Cell 2000, 6(4):947-952.

9. Mishra K, Shore D: Yeast Ku protein plays a direct role in telomeric silencing and counteracts inhibition by rif proteins. Curr Biol 1999, 9 (19):1123-1126.

10. Bourns BD, Alexander MK, Smith AM, Zakian VA: Sir proteins, Rif proteins, and Cdc13p bind Saccharomyces telomeres in vivo. Mol Cell Biol 1998, 18 (9):5600-5608.

11. Smith CD, Smith DL, DeRisi JL, Blackburn EH: Telomeric protein distributions and remodeling through the cell cycle in Saccharomyces cerevisiae. Mol Biol Cell 2003, 14(2):556-570.

12. Kanoh J, Ishikawa F: spRap1 and spRif1, recruited to telomeres by Taz1, are essential for telomere function in fission yeast. Curr Biol 2001, 11 (20):1624-1630.

13. Miller KM, Ferreira MG, Cooper JP: Taz1, Rap1 and Rif1 act both interdependently and independently to maintain telomeres. EMBO J 2005, 24(17):3128-3135.

14. Ramirez-Zavaleta CY, Salas-Delgado GE, De Las Penas A, Castano I: Subtelomeric silencing of the MTL3 locus of Candida glabrata requires yKu70, yKu80, and Rif1 proteins. Eukaryot Cell 2010, 9(10):1602-1611.

15. Adams IR, McLaren A: Identification and characterisation of mRif1: a mouse telomere-associated protein highly expressed in germ cells and embryo-derived pluripotent stem cells. Dev Dyn 2004, 229(4):733-744.

16. Silverman J, Takai H, Buonomo SB, Eisenhaber F, de Lange T: Human Rif1, ortholog of a yeast telomeric protein, is regulated by ATM and 53BP1 and functions in the S-phase checkpoint. Genes Dev 2004, 18(17):2108-2119.

17. Xu L, Blackburn EH: Human Rif1 protein binds aberrant telomeres and aligns along anaphase midzone microtubules. J Cell Biol 2004, 167(5):819-830

18. Buonomo SB, Wu Y, Ferguson D, de Lange T: Mammalian Rif1 contributes to replication stress survival and homology-directed repair. $J$ Cell Biol 2009, 187(3):385-398.

19. Wang H, Zhao A, Chen L, Zhong X, Liao J, Gao M, Cai M, Lee DH, Li J, Chowdhury D, et al: Human RIF1 encodes an anti-apoptotic factor required for DNA repair. Carcinogenesis 2009, 30(8):1314-1319.

20. Xu D, Muniandy P, Leo E, Yin J, Thangavel S, Shen X, li M, Agama K, Guo R Fox $D$ 3rd, et al: Rif1 provides a new DNA-binding interface for the Bloom syndrome complex to maintain normal replication. EMBO J 2010, 29(18):3140-3155.

21. Mason JM, Frydrychova RC, Biessmann H: Drosophila telomeres: an exception providing new insights. Bioessays 2008, 30(1):25-37.

22. Andrade MA, Petosa C, O'Donoghue SI, Muller CW, Bork P: Comparison of ARM and HEAT protein repeats. J Mol Biol 2001, 309(1):1-18.

23. Hendrickx A, Beullens M, Ceulemans H, Den Abt T, Van Eynde A, Nicolaescu E, Lesage B, Bollen M: Docking motif-guided mapping of the interactome of protein phosphatase-1. Chem Biol 2009, 16(4):365-371.

24. Egloff MP, Johnson DF, Moorhead G, Cohen PT, Cohen P, Barford D: Structural basis for the recognition of regulatory subunits by the catalytic subunit of protein phosphatase 1. EMBO J 1997, 16(8):1876-1887. 
25. Liu D, Vleugel M, Backer CB, Hori T, Fukagawa T, Cheeseman IM, Lampson MA: Regulated targeting of protein phosphatase 1 to the outer kinetochore by KNL1 opposes Aurora B kinase. J Cell Biol 2010, 188(6):809-820.

26. Moorhead GB, Trinkle-Mulcahy L, Nimick M, De Wever V, Campbell DG Gourlay R, Lam YW, Lamond Al: Displacement affinity chromatography of protein phosphatase one (PP1) complexes. BMC Biochem 2008, 9:28.

27. Greenwood J, Cooper JP: Non-coding telomeric and subtelomeric transcripts are differentially regulated by telomeric and heterochromatin assembly factors in fission yeast. Nucleic Acids Res 2011, 40(7):2956-2963.

28. Iglesias N, Redon S, Pfeiffer V, Dees M, Lingner J, Luke B: Subtelomeric repetitive elements determine TERRA regulation by Rap1/Rif and Rap1/ Sir complexes in yeast. EMBO Rep 2011, 12(6):587-593.

29. Capkova Frydrychova R, Biessmann H, Mason JM: Regulation of telomere length in Drosophila. Cytogenet Genome Res 2008, 122(3-4):356-364.

30. Torok T, Benitez C, Takacs S, Biessmann H: The protein encoded by the gene proliferation disrupter (prod) is associated with the telomeric retrotransposon array in Drosophila melanogaster. Chromosoma 2007, 116(2):185-195.

31. Darst RP, Garcia SN, Koch MR, Pillus L: Slx5 promotes transcriptional silencing and is required for robust growth in the absence of Sir2. Mol Cell Biol 2008, 28x(4):1361-1372.

32. Martin SG, Laroche T, Suka N, Grunstein M, Gasser SM: Relocalization of telomeric Ku and SIR proteins in response to DNA strand breaks in yeast. Cell 1999, 97(5):621-633.

33. Kennedy BK, Gotta M, Sinclair DA, Mills K, McNabb DS, Murthy M, Pak SM, Laroche T, Gasser SM, Guarente L: Redistribution of silencing proteins from telomeres to the nucleolus is associated with extension of life span in S. cerevisiae. Cell 1997, 89(3):381-391.

34. Park S, Patterson EE, Cobb J, Audhya A, Gartenberg MR, Fox CA: Palmitoylation controls the dynamics of budding-yeast heterochromatin via the telomerebinding protein Rif1. Proc Natl Acad Sci U S A 2011, 108(35):14572-14577.

35. Villasante A, Abad JP, Planello R, Mendez-Lago M, Celniker SE, de Pablos B: Drosophila telomeric retrotransposons derived from an ancestral element that was recruited to replace telomerase. Genome Res 2007, 17(12):1909-1918

36. Anbalagan S, Bonetti D, Lucchini G, Longhese MP: Rif1 supports the function of the CST complex in yeast telomere capping. PLoS Genet 2011, 7(3)::1002024

37. Altschul SF, Madden TL, Schaffer AA, Zhang J, Zhang Z, Miller W, Lipman DJ: Gapped BLAST and PSI-BLAST: a new generation of protein database search programs. Nucleic Acids Res 1997, 25(17):3389-3402.

38. Bailey TL, Williams N, Misleh C, Li WW: MEME: discovering and analyzing DNA and protein sequence motifs. Nucleic Acids Res 2006, 34(Web Server issue):W369-W373.

39. Eddy SR: Profile hidden Markov models. Bioinformatics 1998, 14(9):755.

40. Thompson JD, Gibson TJ, Plewniak F, Jeanmougin F, Higgins DG: The CLUSTAL_X windows interface: flexible strategies for multiple sequence alignment aided by quality analysis tools. Nucleic Acids Res 1997, 25(24):4876.

41. Thompson JD, Higgins DG, Gibson TJ: CLUSTAL W: improving the sensitivity of progressive multiple sequence alignment through sequence weighting, position-specific gap penalties and weight matrix choice. Nucleic Acids Res 1994, 22(22):4673.

42. Tamura K, Dudley J, Nei M, Kumar S: MEGA4: Molecular Evolutionary Genetics Analysis (MEGA) software version 4.0. Mol Biol Evol 2007, 24(8),

43. Walter MF, Biessmann MR, Benitez C, Torok T, Mason JM, Biessmann H: Effects of telomere length in Drosophila melanogaster on life span, fecundity, and fertility. Chromosoma 2007, 116(1):41-51.

doi:10.1186/1471-2164-13-255

Cite this article as: Sreesankar et al: Functional diversification of yeast telomere associated protein, Rif1, in higher eukaryotes. BMC Genomics 2012 13:255.

\section{Submit your next manuscript to BioMed Central and take full advantage of:}

- Convenient online submission

- Thorough peer review

- No space constraints or color figure charges

- Immediate publication on acceptance

- Inclusion in PubMed, CAS, Scopus and Google Scholar

- Research which is freely available for redistribution

Submit your manuscript at www.biomedcentral.com/submit
() Biomed Central 